\title{
Effect of Polyethylene Glycol on Cellulose Acetate Films Designed for Controlled Porosity Osmotic Pump Systems
}

\author{
P. TONGLAIROUM, B PITAKTUNSKUL, T. NGAWHIRUNPAT, P. AKKARAMONGKOLPORN, PRANEET OPANASOPIT AND \\ N. NATTAPULWAT*
}

Department of Pharmaceutical Technology, Faculty of Pharmacy, Silpakorn University, Nakhonpathom 7300, Thailand

Tonglairoum et al.: Effect of Polyethylene Glycol on Cellulose Acetate Films

\begin{abstract}
This study aimed to investigate the properties of the films containing cellulose acetate and different amounts of polyethylene glycol 200 designed for controlled porosity osmotic pump systems. Polyethylene glycol 200 was used as a plasticizer and pore-forming agent and was incorporated into the cellulose acetate films at the varied concentrations. Water permeability through the films was determined based on the osmotic pump system using a device developed for this study. The film properties including polymer interaction, mechanical properties, swelling properties, thickness, porosity, polarity and morphology of the films were investigated. The amount of polyethylene glycol $200 \mathrm{had}$ an effect on the film strength and the water permeability, increasing the amount resulted in greater water permeability. Scanning electron microscopy images showed that there was an increase in the number of pores in the films as the polyethylene glycol 200 content was increased. Moreover, the thickness of the films also affected the water permeation rate, increasing the thickness led to a decrease in the water permeability. This study reported additional knowledge regarding the properties and characteristics of cellulose acetate films with different proportions of polyethylene glycol 200 . This could be applied to the development of a pharmaceutical formulation especially a controlled porosity osmotic pump system in the future.
\end{abstract}

Key words: Cellulose acetate film, water permeability rate, controlled porosity osmotic pump system, polyethylene glycol 200

Generally, drug release from controlled release oral dosage forms would be affected by $\mathrm{pH}$, gastrointestinal motility and the presence of food in gastrointestinal tract. Osmotic controlled-release oral delivery system, which utilizes osmotic pressure as the driving force for controlled delivery of active agent(s), however is not influenced by these factors, and it is possible to predict the release characteristics from the known properties of the drug and the dosage form ${ }^{[1-3]}$. The simplest design of elementary osmotic pump consists of an osmotic core (comprising a drug with or without an osmogent) coated with a semi-permeable membrane. After coming in contact with the gastrointestinal fluids ${ }^{[4,5]}$, it absorbs water at a rate determined by the fluid permeability of the membrane and osmotic pressure of core formulation. This osmotic imbibitions of water results in the formation of a saturated solution of drug within the core, which is dispensed at a controlled rate from the delivery orifice in the membrane ${ }^{[6,7]}$. In addition, there are numerous factors that affect the drug release from osmotic pumps (i.e. drug solubility, osmotic pressure, delivery orifice and types or characteristics of the film). The membrane permeability is one of the important factors that need to be concerned when designing the system because the delivery of drug from oral osmotic systems is controlled by the influx of solvent across the semipermeable membrane, which in turn carries the agent to the outside environment ${ }^{[2,8]}$. Water influx into basic osmotic pump system can be described by the following Eqn. ${ }^{[3]}, \mathrm{dv} / \mathrm{dt}=\mathrm{A} \times \mathrm{Lp} \times(\sigma \Delta \pi-\Delta \mathrm{P}) / \mathrm{h}$, where $d v / d t$ is water influx, $A$ and $h$ are the film area and thickness, respectively; Lp is mechanical

This is an open access article distributed under the terms of the Creative Commons Attribution-NonCommercial-ShareAlike 3.0 License, which allows others to remix, tweak, and build upon the work non-commercially, as long as the author is credited and the new creations are licensed under the identical terms

Accepted 04 December 2018

Revised 30 April 2018

Received 26 June 2017

Indian J Pharm Sci 2019;81(1):117-123 
permeability; $\sigma$ is the reflection coefficient; and $\Delta \pi$ and $\Delta \mathrm{P}$, are the osmotic and hydrostatic pressure difference, respectively, between the inside and outside of the system.

Controlled porosity osmotic pumps are extension of osmotic pump system and are essentially similar, except that there is no need to create a delivery orifice. These systems contain water-soluble additives in the coating membrane; these water-soluble additives dissolve on coming in contact with water, leaving behind pores in the film through which drug release takes place ${ }^{[9]}$. The film is substantially permeable to both water and dissolved solutes and the mechanism of drug release from these systems was found to be primary osmotic, with simple diffusion playing a minor role ${ }^{[10-12]}$. Watersoluble additives that can be used for pore making purpose consist of dimethylsulfone, nicotinamide, saccharides, amino acids, sorbitol, pentaerythritol, mannitol, organic aliphatic and aromatic acids, including diols and polyols, and other water-soluble polymeric materials ${ }^{[10]}$. Polyethylene glycols (PEG) were widely used as plasticizer having a pore forming property to improve film's physical properties and modify drug release characteristics ${ }^{[13]}$. Yang and coworkers studied the influence of different PEG types on the drug release from a monolithic osmotic pump tablet. The result showed that different PEGs had a marked influence on drug release. Comparing to the release through PEG 4000, the release through PEG 400 and PEG 1500 was too fast and the release through PEG 6000 was slower. These authors also studied the influence of the amount of PEG 4000 on drug release profiles. They found that more the PEG 4000 incorporated into the cellulose acetate (CA) film, more was the void space formed after leaching, and as a result, higher the permeability of the film and the release rate obtained. However, high percent of PEG 4000 in CA would make the film fragile ${ }^{[14]}$.

Liu and coworkers ${ }^{[15,16]}$ studied the influence of the nature and the amount of plasticizer on the properties of CA film including drug release profile, thermal properties, microporosity, and mechanical properties. Hydrophilic plasticizer (represented by PEG 200) was found to increase the drug release, whereas hydrophobic plasticizer (represented by triacetin) decreased the drug release from osmotic pumps of nifedipine.

Yuan and coworkers ${ }^{[17]}$ studied the effects of solvent system, PEG molecular weight, and concentration in the films on thermo mechanical properties and permeability of CA-free films. They found that water in the solvent system for film casting can influence the morphology and ultimately the properties of the films. The films prepared from acetone solutions were stronger, tougher and more flexible than the corresponding films made from acetone-water solutions. The PEG level in the film also affects the morphologies. As the PEG concentration increased, the surface smoothness decreased and the pinholes and their size in the cross sections increased.

As mentioned above, the properties of the film or membrane studied is one of the important factors essential for predicting drug release rate from the osmotic pump systems, especially from a controlled porosity osmotic pump system. Therefore, this study aimed to study the properties of a membrane or film used for a controlled porosity osmotic pump system. A device used for investigating water permeation rate through the film was developed based on the osmotic pump system. In this study, PEG 200 was used as a plasticizer as well as a pore-forming agent and incorporated into CA film. The amount of PEG 200 and the thickness of CA membranes were varied. Furthermore, the properties of the film, such as the interaction between CA and PEG 200, mechanical properties, swelling properties, porosity, polarity and morphologies of the film were also evaluated.

\section{MATERIALS AND METHODS}

CA (approximate $40 \%$ acetyl content, CA 39810 NF) was supplied by Eastman Chemical Ltd. (Kingsport, USA). Sodium chloride was obtained from Merck KGaA (Darmstadt, Germany). Polyethylene glycol (PEG, MW 200) was purchased from Sino-Japan Chemical Co., Ltd. (Taipei, Taiwan) and acetone was of analytical grade and was obtained from RCI Labscan Ltd (Bangkok, Thailand).

\section{Preparation of the polymer films:}

Five percent by weight, polymeric solution of CA in acetone were prepared. PEG 200 at the weight ratio of $0,5,10,20,30,40$ or $50 \%$ (based on the polymer weight) was then added into the polymeric solution. The composition of each formulation is presented in Table 1. Each mixture was subsequently cast on a petri dish and left to dry at ambient temperature under clean environment. The dried films were peeled off and stored in desiccators for at least $24 \mathrm{~h}$ prior to further use or evaluation. 
TABLE 1: COMPOSITIONS OF THE CA FILMS

\begin{tabular}{lcc}
\hline $\begin{array}{l}\text { Formulation } \\
\text { (CA:PEG Ratio) }\end{array}$ & $\begin{array}{c}\text { Amount of PEG } \\
\mathbf{2 0 0}\left(\mathbf{m g} / \mathrm{cm}^{2}\right)\end{array}$ & $\begin{array}{c}\text { Amount of CA } \\
\left(\mathbf{m g} / \mathrm{cm}^{2}\right)\end{array}$ \\
\hline F1 (1:0) & 0.00 & 4.60 \\
F2 (1:0.05) & 0.23 & 4.60 \\
F3 (1:0.1) & 0.46 & 4.60 \\
F4 (1:0.2) & 0.92 & 4.60 \\
F5 (1:0.3) & 1.38 & 4.60 \\
F6 (1:0.4) & 1.84 & 4.60 \\
F7 (1:0.5) & 2.30 & 4.60 \\
\hline
\end{tabular}

\section{Evaluation of the polymer films}

The polymer films were characterized for their physicochemical properties as follows: the thickness of the films was measured using an electronic micrometer (Teclock Corporation, Japan). The average of 5 measurement values from 5 pre-set points of each film was calculated. The interaction between CA and PEG 200 was investigated using a Fourier-transform infrared (FTIR) spectrometer (Hyperion 2000, BRUKER, Germany). The FTIR absorption spectrum was recorded at the scanning wave number ranging from 4000 to $500 \mathrm{~cm}^{-1}$.

The mechanical properties of the polymer films were measured using a Texture analyser (Model TA-VXT plus, Stable Microsystems, UK). All samples were cut into rectangle shapes with dimensions of $25 \times 10 \mathrm{~mm}^{2}$. The gauge with $25 \mathrm{~mm}$ in length was used and was driven at a speed of $0.3 \mathrm{~mm} / \mathrm{s}$. The displacement data at rupture were recorded to determine tensile strength $(\mathrm{MPa})$ and elongation values. The averages of five values were calculated. The accurately weighed films were submerged in water for $10 \mathrm{~h}$. After soaking, the film was taken out, wiped with tissue paper and dried at $50^{\circ}$ until a constant weight was obtained. The film thickness, before and after soaking in water was also recorded. The porosity of the film was expressed as percent of weight loss, which was calculated according to the following Eqn. $1^{[18]}$, \% porosity $=(\mathrm{Wb}-\mathrm{Wd}) /$ $\mathrm{Wb} \times 100$, where $\mathrm{Wb}$ and $\mathrm{Wd}$ were the weight of the films before and after soaking in water, respectively. The average of 20 values from each formulation was calculated.

The polarity of the film was determined by measuring the contact angles of 2 testing liquids on the film surface using a First Ten Angstrom device (FTA1000 model, UK) as previously described ${ }^{[19]}$. Purified water, methylene iodide, formamide and ethylene glycol were used as reference solvents. Interfacial surface energy and polarity were recorded. The average of five values from each formula was recorded. Percent polarity of the film was calculated according to the following Eqn. 2, $\%$ polarity $=$ polar $/$ IFT $($ energy $) \times 100$.

The surface morphology of the film was determined using scanning electron microscopy (JSM-5410 LV, Jeol, Tokyo, Japan). A small section of the film was sputtered with a thin layer of gold before the SEM observations.

Self-adapted water permeability testing instrument was designed as shown in fig. 1. The instrument consists of two main compartments, which contained saturated sodium chloride in one compartment (A) and deionized water in another compartment (B). Free film was mounted between both compartments (C). Whole system was surrounded with double jackets (E) at controlled temperature of $37 \pm 0.5^{\circ}$. Small glass pipes were attached to each compartment and connected with a weighing balance $(\mathrm{G})$ via peristaltic pump $(\mathrm{F})$. The weight of water permeated from compartment $\mathrm{B}$ to compartment A was recorded every 10 min for $12 \mathrm{~h}$. The water permeability of the film was determined by the rate of water permeation through the film. At the end of the permeation testing, the tested film was dried in an oven. The FTIR spectrum, mechanical properties and morphologies of the dried film were evaluated.

\section{RESULTS AND DISCUSSION}

The components used for the preparation of semipermeable membrane or film in an osmotic pump system should be compatible ${ }^{[20]}$. FTIR is an efficient and rapid analytical technique used to identify the interaction between substances ${ }^{[21]}$. In this study, FTIR was applied to evaluate the interaction between the CA films that were cast with acetone as a solvent and PEG 200, which was used as a pore-forming agent. Fig. 2 displays the FTIR spectra of acetone, CA powder and CA film (F1), respectively. The results revealed that the FTIR spectrum of F1 was similar to the spectrum of CA powder, which exhibited a dominant

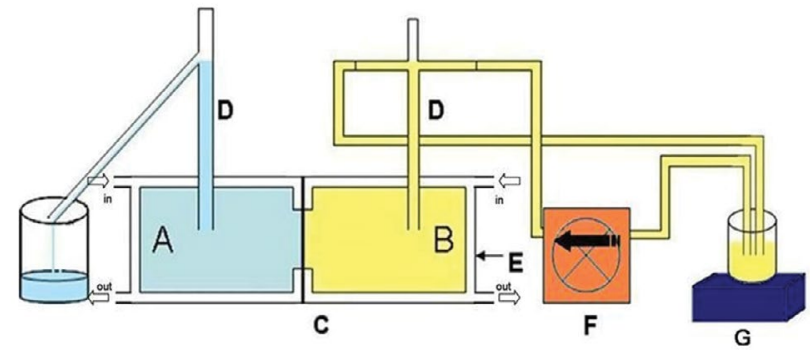

Fig. 1: Design of water permeability testing instrument (A) saturated sodium chloride, (B) deionized water, (C) free film, (D) glass tubes, (E) double jackets, (F) peristaltic pump, and $(G)$ weighing balance 
peak of esters group at the wave number of $1740 \mathrm{~cm}^{-1}$. The results indicated that acetone, a solvent used for CA film preparation, did not affect the structure of CA at molecular level. Meanwhile, the broad spectra of alcohol groups were observed at the wave number of $3368 \mathrm{~cm}^{-1}$, and the intensities were found to be increased as the amount of PEG 200 in the films increased as presented in fig. 3. However, the dominant peaks in the spectra of CA film were not changed. These results suggested that there was no interaction at the molecular level between PEG 200 and CA in the films.

The semi-permeable membrane or film in osmotic pump systems must be sufficiently rigid so as to retain its dimensional integrity during the operational lifetime of the devices ${ }^{[22]}$. The mechanical properties of the films were measured by tensile strength and percentage of elongation. The tensile strength reflects the energy required to break the film by stretching. On the other hand, the percentage of elongation data indicated the extent to which the films could be stretched. The results showed that after increasing the amount of PEG 200 in the films, a decrease in the tensile strength had been observed. This might be due to the plasticization of the polymer. Increasing the PEG 200 concentration from 5 to $30 \%$ resulted in a steep increase in the elongation at rupture of the films. At the higher levels of PEG 200, the elongation was found to be decreased as a result of phase separation of PEG and CA. This was in accordance with the study of Wang et al. ${ }^{[23]}$. The tensile strength and elongation at rupture of the films containing different amounts of PEG 200 was presented in figs. $4 \mathrm{~A}$ and $\mathrm{B}$, respectively.

Porosity and swelling properties are the factors affecting the water permeability of CA film, if CA films swell and extend, water will have an impact on the

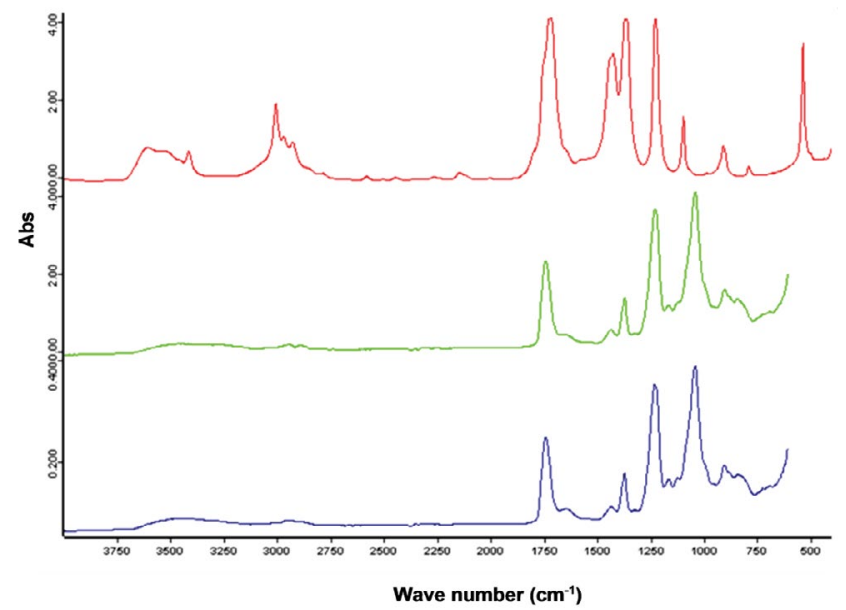

Fig. 2: FT-IR Spectra of acetone, CA powder and CA film (F1) Acetone; C CA powder; — CA film (W1)
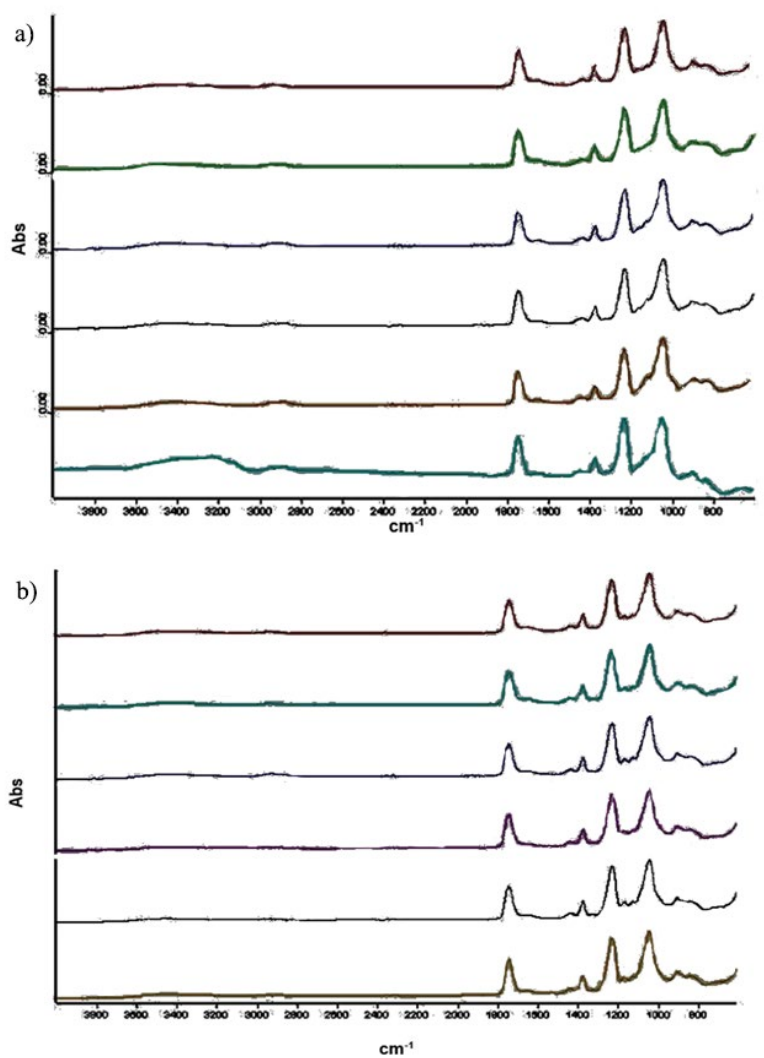

Fig. 3: IR spectra of the CA films

IR spectra of the $C A$ films containing $C A$ and different amounts of PEG 200. (a) Before water permeation test, - F2, - F3, F4, - F5, - F6, - F7 (b) after water permeation test, - F2, - F3, - F4, - F5, - F6, - F7. Films containing CA and different amounts of PEG 200: F2 (5\%), F3 (10\%), F4 (20 $\%)$, F5 (30 \%), F6 (40 \%) and F7 (50\%) w/w with respect to the weight of $\mathrm{CA}$

thickness of the films, and the thickness will affect the permeability of water through the films. To study these properties, the CA films with different thicknesses and different amounts of PEG 200 (F1 to F7) were employed for the investigation. After determining the thickness of film before and after the experiment, the results indicated that the films did not swell and the thickness and the weight of the films decreased with respect to the amount of PEG 200 in the films (Table 2). This could be because of the very good water solubility of PEG 200 leading to the dissolution of the film during the experiment. As a result, pores were generated after the PEG 200 dissolved from the films. The \% porosity of the films increased as the amount of PEG in the films increased as shown in Table 2.

The polarity of the films could be determined by measuring the contact angles of two testing liquids on the film surface using a First Ten Angstrom device. The results revealed that the \% polarity of the film depended on the amount of PEG 200 contained in the 

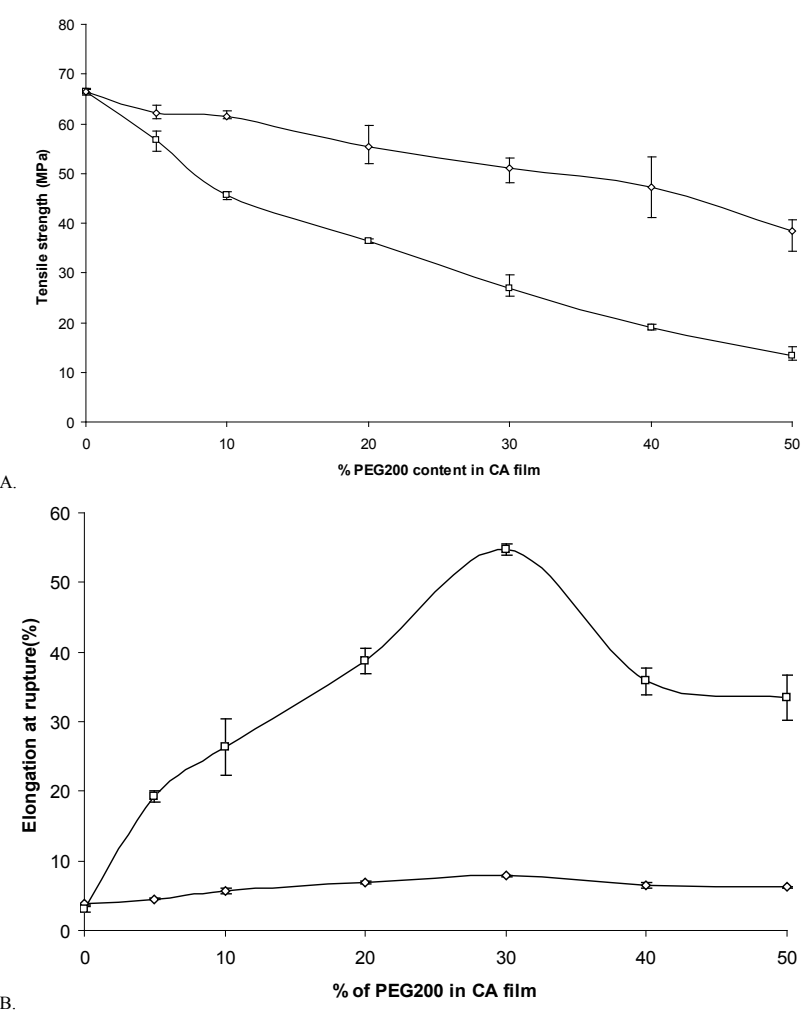

Fig. 4: Tensile strength and percent elongation at rupture of the CA films

(A) Tensile strength and (B) percent elongation at rupture of the CA films containing different amounts of PEG 200. (-口-) Before water permeation test, $\left(-\nabla_{-}\right)$after water permeation test

TABLE 2: POROSITY AND DECREASE IN THICKNESS OF THE CA FILMS

\begin{tabular}{lcc}
\hline Formula & \% Porosity & \% Decrease in thickness \\
\hline F1 & $0.20 \pm 0.295$ & $0.09 \pm 0.28$ \\
F2 & $0.99 \pm 0.850$ & $0.38 \pm 0.71$ \\
F3 & $9.63 \pm 0.372$ & $0.89 \pm 0.86$ \\
F4 & $19.52 \pm 0.432$ & $1.91 \pm 0.65$ \\
F5 & $29.65 \pm 0.371$ & $2.94 \pm 0.40$ \\
F6 & $39.42 \pm 0.382$ & $4.94 \pm 0.59$ \\
F7 & $49.12 \pm 0.562$ & $9.56 \pm 1.17$ \\
\hline
\end{tabular}

Percent porosity and \% decrease in thickness of the CA films containing different amounts of PEG 200 after being soaked in di water for $10 \mathrm{~h}$

film as displayed in Table 3. In fact, the polarity value depended on the quantity of hydroxyl group in the film, which was correlated with the amount of \% PEG 200 contained in the film.

The porosity and structure of the films were examined using SEM. Fig. 5 shows the SEM images of the films prepared from F1, F2, F3, F4, F5, F6 and F7, respectively. It could be observed that the film fabricated without PEG 200 exhibited a smooth surface without pore. The addition of PEG 200 led to a difference in film morphologies as displayed in fig. 5B, C, D, E, F and $\mathrm{G}$. The surface of the film with $50 \%$ PEG 200 became significantly more porous and rough. PEG 200 might facilitate the phase inversion of the polymer solution. Faster phase inversion induced by the amount of PEG in the film led to the more porous film structures ${ }^{[24]}$.

Water permeability through fabricated CA films could be determined using a water permeability testing apparatus as presented in fig. 1. This apparatus was designed based on the permeability with osmotic pressure principle. As shown in fig. 1, compartment A contained saturated sodium chloride solution, which was an osmogent that generates osmotic pressure. Meanwhile, compartment B comprised distilled water and was connected with a tube (D), this tube sucked water up from the beaker on weighing balance through peristaltic pump until the level of water in two compartments were equal. Afterwards, distilled water from compartment $\mathrm{B}$ would pass through $\mathrm{CA}$ film (diameter $2.5 \mathrm{~cm}$ ) to compartment $\mathrm{C}$, and then to compartment $\mathrm{A}$. Then, the weight loss of water in part $G$ over that time was recorded. From this study, the CA film without PEG 200 as a pore-forming agent (F1), water could slightly permeable through CA film in all thicknesses. The increase in water permeability rate had been observed with an increase in the amount of PEG 200 in the CA film (Table 4). Furthermore, the thickness of the films also had an effect on the water permeability rate. The thicker films demonstrated lower water permeability rate as displayed in fig. 6. This result correlated well with that obtained in a previous study ${ }^{[24,25]}$.

The film interaction was investigated at the end of water permeation test, and the results are shown in fig. 3B. It can be observed from the FTIR spectra that PEG 200 was eliminated from the film prepared from F2, F3, F4, F5, F6 and F7 after the water permeation test. The films exhibited similar spectra with the CA film.

The tensile strength and elongation properties of films were also assessed at the end permeation test and the

TABLE 3: POLARITY OF THE CA FILMS

\begin{tabular}{ll}
\hline Formula & \% Polarity \\
\hline F1 & $27.34 \pm 0.51$ \\
F2 & $28.34 \pm 0.30$ \\
F3 & $29.82 \pm 0.46$ \\
F4 & $33.26 \pm 0.21$ \\
F5 & $38.00 \pm 0.17$ \\
F6 & $40.28 \pm 0.40$ \\
F7 & $41.69 \pm 0.41$ \\
\hline
\end{tabular}


a
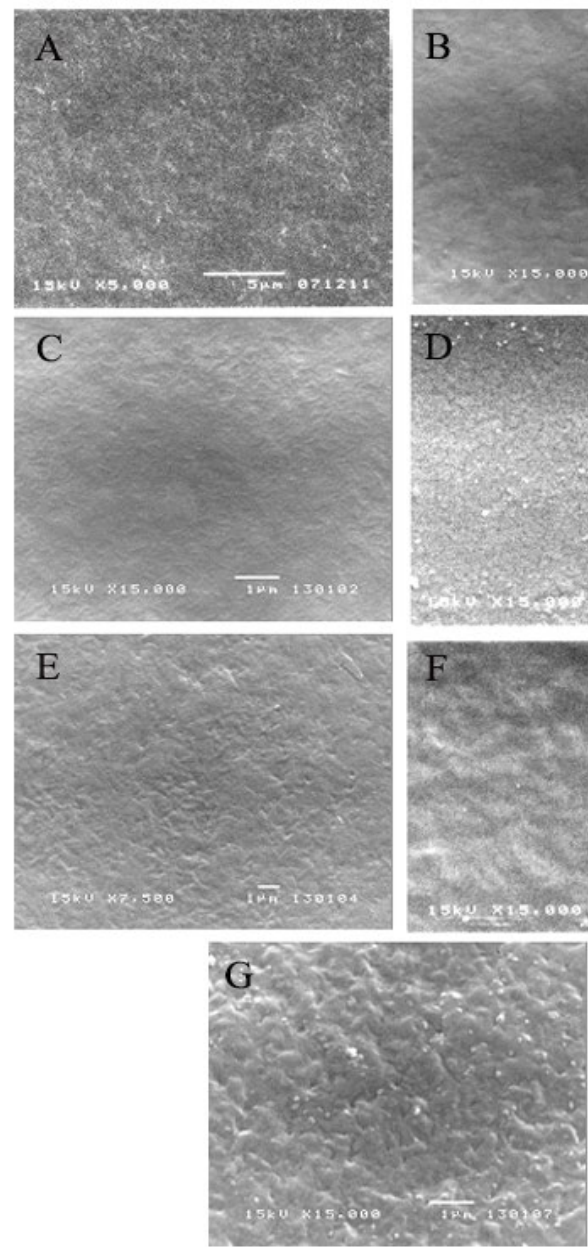

b
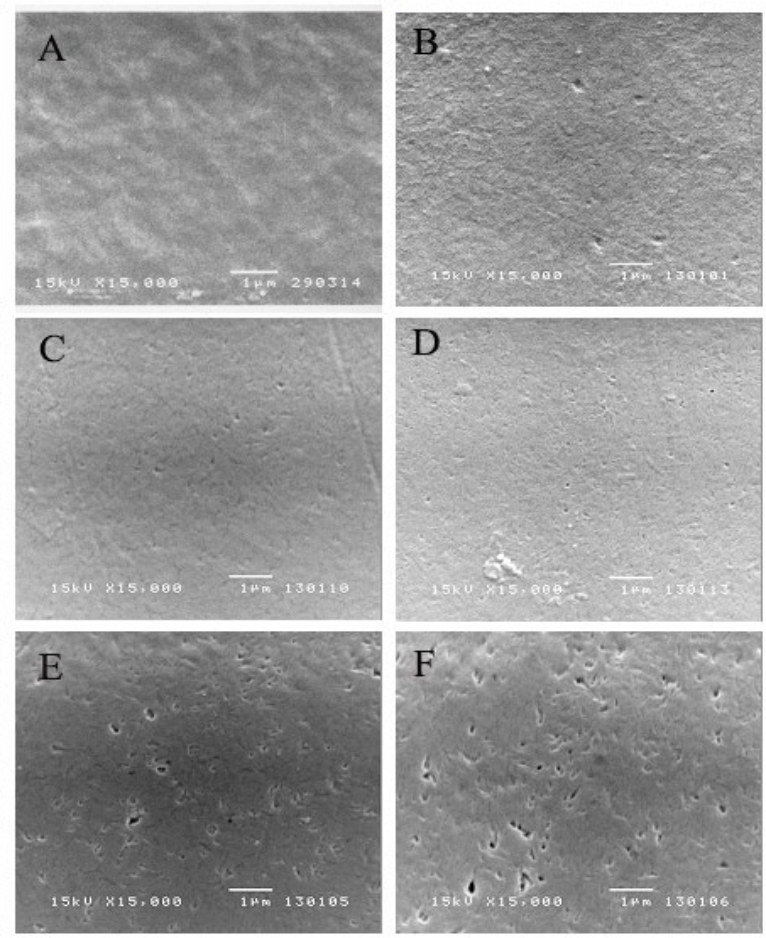

$\mathrm{D}$

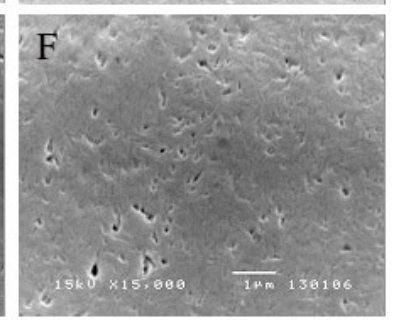

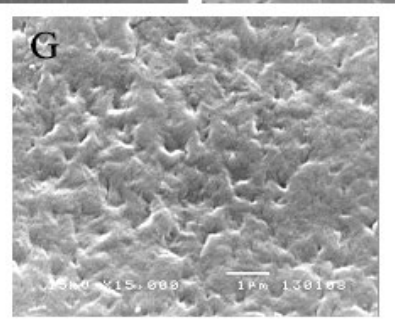

Fig. 5: SEM images of the CA films containing different amounts of PEG 200

SEM images of the CA films containing different amounts of PEG 200 (a) before and (b) after water permeation test. (A) F1, (B) F2, (C) F3, (D) F4, (E) F5, (F) F6, (G) F7

TABLE 4: WATER PERMEABILITY RATE OF THE CA FILMS

\begin{tabular}{lcc}
\hline Formula & Permeability rate $\left.\mathrm{gm} / \mathrm{min} / \mathrm{mm}^{2}\right)$ & $\mathrm{R}^{2}$ \\
\hline F1 & $1.00 \times 10^{-6}$ & 0.9229 \\
F2 & $1.00 \times 10^{-4}$ & 0.9873 \\
F3 & $2.00 \times 10^{-4}$ & 0.9330 \\
F4 & $2.00 \times 10^{-4}$ & 0.9837 \\
F5 & $3.00 \times 10^{-4}$ & 0.9363 \\
F6 & $4.00 \times 10^{-4}$ & 0.9824 \\
F7 & $5.00 \times 10^{-4}$ & 0.9974 \\
\hline
\end{tabular}

Water permeability rate was tested by a water permeability testing equipment

results are presented in fig. $4 \mathrm{~A}$ and $\mathrm{B}$, respectively. The tensile strength of the films (F2, F3, F4, F5, F6 and F7) was decreased. This may be because the films became more porous after losing PEG 200 from the films. However, the percentage of elongation at rupture of the films was not evidenced after the PEG was eliminated.
This result was in accordance with the study of Wang et al. ${ }^{[23]}$.

The morphology of the films at the end of permeation test was determined and the results are displayed in fig. 5, as could be seen from fig. 5, there was an increase in the number of pores in the films as the PEG 200 content increased. This result related well with the porosity study mentioned above. Additionally, the pore sizes occurred on the films was less than $1 \mu \mathrm{m}$. Thus, the films with similar thickness that contained different amount of PEG 200 could make a difference in water permeability rate. The pores can be continuous pores which open at both side of the microporous lamina pore interconnected through tortuous paths of regular and irregular shapes ${ }^{[9]}$.

This study examined the impacts of PEG 200 concentrations as a pore-forming agent on the CA film by systematically evaluating the interaction between 


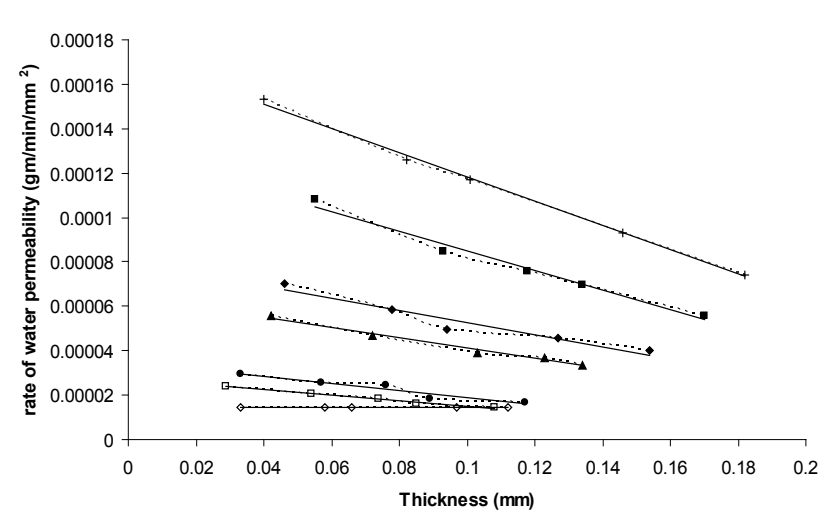

Fig. 6: Water permeability of the CA films Water permeability profiles of the $\mathrm{CA}$ films containing different

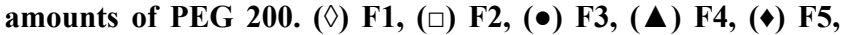
(घ) F6, (+) F7

a CA film and PEG 200 as well as the mechanical properties, porosity, swelling properties, and water permeability rates of the CA film containing different amount of PEG 200. This study revealed that the amount of PEG 200 in the film affected the structure and strength of the CA films. Additionally, the amount of PEG 200 in the CA films had an impact on the water permeation rate. The knowledge obtained from this study can assist in formulating a drug delivery system such as controlled porosity osmotic pump systems that employs CA film as a component and contains PEG 200 as a plasticizer or pore-forming agent for the films.

\section{Acknowledgements:}

The authors would like to thank the Faculty of Pharmacy, Silpakorn University for financial support.

\section{Conflict of interest:}

The authors report no conflicts of interest in this work.

\section{REFERENCES}

1. Theeuwes F, Swanson DR, Guittard G, Ayer A, Khanna S. Osmotic Delivery Systems for the Beta-Adrenoceptor Antagonists Metoprolol and Oxprenolol: Design and Evaluation of Systems for Once-Daily Administration. Br J Clin Pharmacol 1985;19(2):69S-76S.

2. Verma RK, Mishra B, Garg S. Osmotically controlled oral drug delivery. Drug Dev Ind Pharm 2000;26(7):695-708.

3. Theeuwes F. Elementary osmotic pump. J Pharm Sci 1975;64(12):1987-91.

4. Khan ZA, Tripathi R, Mishra B. Floating elementary osmotic pump tablet (FEOPT) for controlled delivery of diethylcarbamazine citrate: a water-soluble drug. AAPS PharmSciTech 2011;12(4):1312-23.

5. Gupta BP, Thakur N, Jain NP, Banweer J, Jain S. Osmotically controlled drug delivery system with associated drugs. J Pharm Pharm Sci 2010;13(4):571-88.
6. Keraliya RA, Patel C, Patel P, Keraliya V, Soni TG, Patel RC, et al. Osmotic Drug Delivery System as a Part of Modified Release Dosage Form. ISRN Pharm 2012;2012:528079.

7. Verma RK, Krishna DM, Garg S. Formulation aspects in the development of osmotically controlled oral drug delivery systems. J Control Release 2002;79(1-3):7-27.

8. Verma RK, Arora S, Garg S. Osmotic pumps in drug delivery. Crit Rev Ther Drug Carrier Syst 2004;21(6):477-520.

9. Haslam JL, Rork GS, inventor; Merck and Co Inc., assignee. Controlled porosity osmotic pumps. United States patent US4880631A. 1989 Nov 14.

10. Zentner GM, Rork GS, Himmelstein KJ, inventor; Merck and Co Inc., assignee. Controlled porosity osmotic pump. United States patent US4968507A. 1990 Nov 6.

11. Zentner GM, Rork GS, Himmelstein KJ. The controlled porosity osmotic pump. J Control Release 1985;1(4):269-82.

12. Zentner GM, Rork GS, Himmelstein KJ. Osmotic flow through controlled porosity films: An approach to delivery of water soluble compounds. J Control Release 1985;2:217-29.

13. Zhu Y, Mehta KA, McGinity JW. Influence of plasticizer level on the drug release from sustained release film coated and hot-melt extruded dosage forms. Pharm Dev Technol 2006;11(3):285-94.

14. Yang XG, Zhang GH, Li W, Peng B, Liu ZD, Pan WS. Design and evaluation of jingzhiguanxin monolithic osmotic pump tablet. Chem Pharm Bull 2006;54(4):465-9.

15. Liu L, Khang G, Rhee JM, Lee HB. Preparation and characterization of cellulose acetate membrane for monolithic tablet. Korea Polym J 1999;7(5):289-96.

16. Rao PR, Diwan PV. Permeability studies of cellulose acetate free films for transdermal use: Influence of plasticizers. Pharm Acta Helv 1997;72(1):47-51.

17. Yuan J, Shang PP, Wu S. Effects of polyethene glycol on morphology, thermomechanical properties and water vapour permeability of cellulose acetate free films. Pharm Technol North Am 2001;25(10):62.

18. Chakrabarty B, Ghoshal AK, Purkait MK. Effect of molecular weight of PEG on membrane morphology and transport properties. J Memb Sci 2008;309(1-2):209-21.

19. Wu S, Brzozowski KJ. Surface free energy and polarity of organic pigments. J Colloid Interface Sci 1971;37(4):686-90.

20. Mathur M, Mishra R. A Review on Osmotic Pump Drug Delivery System. Int J Pharm Sci Res 2016;7(2):453-71.

21. Lin PC, Lin S, Wang PC, Sridhar R. Techniques for physicochemical characterization of nanomaterials. Biotechnol Adv 2014;32(4):711-26.

22. Sahoo CK, Sahoo NK, Rao SRM, Sudhakar M, Satyanarayana KA. Review on controlled porosity osmotic pump tablets and its evaluation. Bull Fac Pharm Cairo Univ 2015;53(2):195205.

23. Wang FJ, Yang YY, Zhang XZ, Zhu X, Chung TS, Moochhala S. Cellulose acetate membranes for transdermal delivery of scopolamine base. Mater Sci Eng C Mater Biol Appl 2002;20(1-2):93-100.

24. Kim JH, Lee KH. Effect of PEG additive on membrane formation by phase inversion. J Memb Sci 1998;138(2):15363.

25. Verma RK, Krishna DM, Garg S. Formulation aspects in the development of osmotically controlled oral drug delivery systems. J Control Release 2002;79(1-3):7-27. 